\title{
PLANEJAMENTO ESTRATÉGICO SITUACIONAL Relato de uma Experiência em uma Farmácia Municipal do Sul do Brasil
}

\author{
Cristiano Manetti ${ }^{1}$ \\ Márcia dos Angeles Luna Leite ${ }^{2}$
}

\begin{abstract}
RESUMO
Este texto tem por objetivo relatar a experiência de elaboração de um Plano Operativo (P0), desenvolvido com a finalidade de qualificar a Assistência Farmacêutica (AF) da Farmácia Municipal de Canguçu, Rio Grande do Sul. Trata-se de um relato e uma reflexão sobre uma atividade prática e colaborativa, com vistas à aplicação futura na Farmácia Municipal. Os autores deste relato não se colocam apenas como observadores, mas como propositores e membros da equipe que elaborou o plano de intervenção na realidade da AF do município. São apresentados os passos da elaboração de um P0, visando proporcionar direcionalidade à gestão a fim de intervir em uma realidade sobre a qual não é possível ter total controle. As informações expostas neste artigo foram obtidas nas oficinas de construção do P0 e em referências bibliográficas. As oficinas, divididas em quatro etapas, contaram com a participação de diferentes atores que trabalham na prefeitura de Canguçu, em funções relacionadas ao objetivo desse estudo. Após a elaboração do PO houve a constatação de que a desatualização da Relação Municipal de Medicamentos Essenciais, que não estava de acordo com o perfil epidemiológico dos cidadãos canguçuenses, tem elevado o número de processos judiciais contra o Estado e/ou município nos últimos anos. Espera-se que o presente estudo possa contribuir para a qualificação da AF na Atenção Básica do município, fomentando o uso diário da ferramenta Planejamento Estratégico Situacional para a identificação de problemas, com a participação dos diversos atores envolvidos no atendimento da Secretaria Municipal de Saúde.
\end{abstract}

Palavras-chave: Planejamento Estratégico Situacional. Relato de experiência. Serviços comunitários de farmácia.

SITUATIONAL STRATEGIC PLANNING: REPORT OF AN EXPERIENCE IN A MUNICIPAL PHARMACY IN SOUTHERN BRAZIL. ABSTRACT

This work aims to report the development of an Operating Plan experience (OP), developed for the purpose of qualifying the Pharmaceutical Care (PC) of the Municipal Pharmacy of Canguçu, Rio Grande do Sul. It is a report and a reflection on a practical and collaborative activity with a view to future investment in Municipal Pharmacy. The authors of this report does not arise only as observers, but as proposers and members of the team that prepared the action plan in PC reality of the city. The construction steps are presented of an OP, which aims to provide directionality to the management, in this case Pharmaceutical Assistance, in order to intervene in a reality in which you cannot have full control. The information presented in this article were obtained through the OP building workshops and bibliography references. Workshops divided into four stages, with the participation of different actors working in the City Hall of Canguçu in functions related to the objective of this study. After the development of $\mathrm{OP}$, it was realized that the downgrade of Municipal Relation of Essential Medicines of the Municipal Pharmacy of Canguçu/RS, which is not according to the epidemiological profile of the citizens, it is raising the number of lawsuits against the state and/or the city. It is hoped that this study may contribute to the qualification of PC in Primary Care of the city with the everyday use of Situational Strategic Planning Tool in identifying problems with the participation of the various actors involved in the attendance of the Municipal Health Care.

Keywords: Situational Strategic Planning. Report of an experience. Community Pharmacy services.

\footnotetext{
${ }^{1}$ Especialista em Gestão da Assistência Farmacêutica pela Universidade Federal de Santa Catarina. Especialista em Farmácia Clínica-Farmacologia pela Universidade Católica de Pelotas. Graduado em Farmácia e Bioquímica com Ênfase em Análises Clínicas pela Universidade Católica de Pelotas. Graduado em Licenciatura Plena na Área da Saúde pela Universidade Católica de Pelotas. Farmacêutico responsável técnico pela Farmácia Municipal de Canguçu/RS. cristiano_manetti@yahoo.com.br

2 Doutora em Ciências Farmacêuticas pela Universidade Federal do Rio Grande do Sul. Mestre em Ciências Farmacêuticas pela Universidade Federal do Rio Grande do Sul. Graduada em Farmácia pela Universidade Federal do Rio de Janeiro. marcia.angeles@gmail.com
} 
Desde a criação da Organização Mundial da Saúde (OMS), os medicamentos são considerados insumos essenciais para que se alcance o nível máximo de saúde. A partir da Conferência Mundial sobre Atenção Primária à Saúde (APS), em 1978, foi proposta a criação de uma lista de medicamentos essenciais para ser seguida como modelo, de forma flexível, levando em consideração as necessidades de cada país (Organização Pan-Americana da Saúde, Organização Mundial da Saúde, 2013).

A Política Nacional de Assistência Farmacêutica (PNAF), aprovada pelo Conselho Nacional de Saúde em 2004, por meio da resolução $n^{\circ} 338$, tem por base princípios que tratam da Assistência Farmacêutica (AF) como parte integrante da Política Nacional de Saúde (PNS).

A PNAF possui entre seus eixos estratégicos a necessidade de manutenção da assistência farmacêutica na rede pública, a qualificação dos serviços, a capacitação de recursos humanos, a promoção do uso racional de medicamentos e a utilização da Relação Nacional de Medicamentos Essenciais (Rename) como instrumento racionalizador.

O principal acesso ao sistema de assistência à saúde no Brasil ocorre nas Unidades Básicas de Saúde (UBS) e, nesses estabelecimentos, normalmente as farmácias ocupam espaços relativamente pequenos e as práticas reforçam a relação da $\mathrm{AF}$ com o modelo curativo "centrado na consulta médica e pronto atendimento, com a farmácia apenas atendendo a essas demandas" (Araújo et al., 2008). Essa configuração prejudica a atuação do farmacêutico em relação à orientação dos usuários, interferindo em um trabalho que é imprescindível para que haja uma melhor adesão do usuário e para reduzir possíveis problemas relacionados ao uso inadequado dos medicamentos.

Com o objetivo de promover a educação permanente de farmacêuticos e qualificar a AF do SUS, o Ministério da Saúde (MS) estabeleceu uma parceria com a Universidade Federal de Santa Catarina (UFSC) e ampliou a qualificação nacional de farmacêuticos por meio do curso "Gestão da Assistência Farmacêutica - Especialização a Distância”. A es- tratégia de ensino-aprendizagem do curso baseou-se na construção de um Plano Operativo (PO) com foco na gestão da AF.

O Planejamento Estratégico Situacional (PES) foi concebido pelo economista chileno Carlos Matus, a partir de sua vivência como ministro da Economia no governo Allende, entre 1970 e 1973 . O PES é um método de planejamento orientado por problemas e trata, principalmente, daqueles que são mal-estruturados e complexos, para os quais não existe solução normativa ou previamente conhecida como no caso de problemas bem estruturados. É importante destacar que, embora se possa partir de um campo ou setor específico, os problemas são sempre abordados em suas múltiplas dimensões política, econômica, social, cultural, etc. - e em sua multissetorialidade, pois suas causas não se limitam ao interior de um setor ou área específicos e sua solução depende, muitas vezes, de recursos extrassetoriais e da interação dos diversos atores envolvidos na situação (Artmann, 2000).

O PES, de acordo com Rivera e Artmann (1999), surgiu na década de 70 com a ideia de introduzir o diálogo na atividade de planejamento, articulando sujeitos sociais e propondo uma problematização coletiva visando a uma construção de projeto assumida por todos, priorizando a comunicação. Para os autores, o modelo de organização formulado por Carlos Matus deve ser reflexivo como condição de governabilidade, além da necessidade de construção de indicadores para o monitoramento do plano. Segundo Matus, um ator com conhecimento do problema e capacidade de raciocínio estratégico com certeza enfrentará melhor um dilema do que alguém com baixo conhecimento da realidade e apenas conhecimento de método, por melhor que este seja (Rivera; Artmann, 1999, p. 364).

O SUS foi criado pela Constituição Federal de 1988 e regulamentado pela Lei 8.080/90 que documenta, em seu artigo 196, que a saúde é um direito de todos e dever do Estado, logo tem como princípios a equidade, a universalidade e a integralidade.

A AF foi regulamentada no âmbito do SUS em 1998, com a criação da Política Nacional de Medicamentos (PNM), contudo foi reorientada poste- 
riormente pela I Conferência Nacional de Medicamentos e Assistência Farmacêutica em 2003 e pela PNAF em 2004. Somente em 2006, com a publicação dos Pactos pela Vida e de Gestão, a AF passou a contar com um bloco de financiamento específico no SUS (Pepe et al., 2010).

Posteriormente, com a criação e atualização regular da Rename, os municípios e Estados passaram a contar com uma lista de fármacos que inclui os componentes básico, estratégico e especializado da AF. Com isso há a promoção do acesso e o uso racional da medicação.

A gestão do SUS é descentralizada entre os governos federal, estaduais e municipais. Devido às dimensões do país e ao perfil epidemiológico da população brasileira, há uma orientação para que cada governo selecione quais os medicamentos que irão compor a sua lista local ou regional (Brasil, 2001). A Relação Municipal de Medicamentos (Remume) é baseada na Rename, contudo deve ser adaptada ao perfil de morbimortalidade do município.

Pesquisas científicas que abordam a judicialização na AF apontam para o aumento da demanda judicial, fazendo com que os municípios tenham de comprar medicamentos que não fazem parte da Rename ou Remume. Alguns desses fármacos não possuem registro na Agência Nacional de Vigilância Sanitária (Anvisa) e muitos são prescritos por profissionais da rede privada, causando prejuízos aos princípios do SUS: equidade, integralidade e universalidade, agravando com isto o uso irracional de medicamentos e aumento do uso de recursos financeiros públicos (Vieira; Zucchi, 2007).

No município de Pelotas/RS foi elaborado um estudo sobre a judicialização de processos na $3^{\mathrm{a}}$ Coordenadoria Regional de Saúde, com dados obtidos por meio do sistema Administração de Medicamentos do Estado (AME). Esta Coordenadoria é responsável por 22 municípios da Região Sul do Estado do RS, incluindo Canguçu. Em janeiro de 2012 estavam cadastrados neste sistema 3.714 ações judiciais, das quais $45 \%$ eram de usuários residentes em Pelotas, totalizando 4.360 medicamentos solicitados. Destes, $43 \%$ não eram padronizados pelo SUS, 19\% eram de competência do município e 38\% estavam na lista de medicamentos fornecidos pelo Estado. Foram levantadas como causas da judicialização as dificuldades de gestão da AF, o elevado número de usuários do SUS e a extensão territorial do município, fazendo com que o gestor encontrasse dificuldades de interlocução com os usuários (Fredes, 2012).

Este artigo tem o objetivo de relatar a experiência de elaboração de um Plano Operativo, parte integrante do Planejamento Estratégico Situacional, desenvolvido com a finalidade de qualificar a Assistência Farmacêutica da Farmácia Municipal de Canguçu/RS.

\section{FOCO DO ESTUDO}

O município de Canguçu localiza-se na Região Sul do Rio Grande do Sul (RS), a aproximadamente $56 \mathrm{~km}$ de Pelotas e $274 \mathrm{~km}$ de Porto Alegre. De acordo com o Instituto Brasileiro de Geografia e Estatística (IBGE), em 2013 Canguçu possuía 53.259 habitantes, com a maior parte da população residindo na zona rural. Conforme o Atlas Brasil (2010), que divulga dados do Instituto de Pesquisa Econômica Aplicada (Ipea), 63,2\% da população de Canguçu, em 2010, residia na zona rural do município. No mesmo ano, $50,43 \%$ dos habitantes eram homens, $67,81 \%$ da população possuía entre $15 \mathrm{e}$ 64 anos e apenas $28,73 \%$ dos habitantes entre $18 \mathrm{e}$ 20 anos possuíam Ensino Médio completo.

Por ocasião do estudo, a Atenção Básica em Canguçu estava composta por: um Pronto Atendimento (PA) 24 horas; um Posto de Saúde Central (PSC), responsável pelos atendimentos odontológico, pediátrico, nutricional, vacinação, Doenças Sexualmente Transmissíveis (DSTs) e Síndrome da Imunodeficiência Adquirida (Aids), vigilância epidemiológica e grupos de usuários hipertensos e diabéticos (Hiperdia); dois Centros de Apoio Psicossociais (Caps), Caps AD para usuários de álcool e drogas e Caps I para indivíduos com transtornos mentais severos e persistentes; uma farmácia municipal; um Centro de Especialidades Odontológicas (CEO); uma unidade de Serviço de Atendimento 
Móvel de Urgência (Samu); 21 Unidades Básicas de Saúde localizados no interior do município e uma unidade móvel com atendimento diário nas localidades do interior, mas distantes das Unidades de Saúde.

A Atenção Básica nos cinco subdistritos de Canguçu estava então dividida da seguinte forma: duas UBS no primeiro distrito; cinco UBS no segundo distrito; seis UBS no terceiro distrito; cinco UBS no quarto distrito; três UBS no quinto distrito; cinco Unidades de Saúde da Família (USF), três delas localizadas na cidade e duas na área rural do município.

Na Farmácia Municipal centrava-se a maior parte da dispensação de medicamentos fornecidos pelo município e Estado, que compõem respectivamente a lista básica da Rename e o componente especializado da Assistência Farmacêutica. A Remume não era utilizada como base para a aquisição e dispensação porque estava desatualizada desde 2006. A entrega de medicamentos para hipertensão e diabetes nos grupos do Sistema de Cadastramento e Acompanhamento de Hipertensos e Diabéticos (Hiperdia) nas USF na área urbana e rural do município ocorria em Unidades de Saúde que não contavam com a presença de um farmacêutico.

Além dos medicamentos da lista básica do município baseada na Rename de 2014, conforme o artigo 26 da Portaria Ministerial 204/2007, os usuários do SUS contavam com a lista de medicamentos do componente estratégico para: hanseníase, doença de Chagas, tuberculose, malária, leishmaniose e outras doenças endêmicas de abrangência nacional e regional, antirretrovirais do programa DST/Aids, sangue e hemoderivados e imunobiológicos (Brasil, 2007). Conforme a Portaria 1.554, de 30 de julho de 2013, também eram disponibilizados os fármacos que fazem parte do componente especializado da Assistência Farmacêutica (Brasil, 2013). Para encaminhamento de processos administrativos ao Estado do RS para obtenção de fármacos de alta complexidade, os usuários devem preencher os requisitos do Protocolo Clínico e Diretrizes Terapêuticas (Rio Grande do Sul, 2015) e dos medicamentos especiais (Rio Grande do Sul, 2014), contudo estes são fornecidos somente pela Secretaria Estadual de Saúde do RS.

O município contava com apenas dois profissionais para atender toda a demanda de serviços farmacêuticos na Secretaria Municipal de Saúde (SMS).

No prédio da Farmácia Municipal também se localizava a Central de Abastecimento Farmacêutico (CAF), em que atuava um farmacêutico responsável também pela compra, conferência e distribuição de medicamentos para as UBSs do município. Da área física da Farmácia Municipal, de aproximadamente $100 \mathrm{~m}^{2}$, quase $30 \%$ estava reservada ao CAF.

A Farmácia Municipal de Canguçu/RS foi escolhida como foco do planejamento por ser o local onde um dos autores deste artigo atua como Responsável Técnico desenvolvendo ações como: o encaminhamento e a dispensação de medicamentos do Componente Especializado da Assistência Farmacêutica, a dispensação de fraldas, a gestão do Componente Estratégico, controle de insulinas e fitas reagentes para glicose, encaminhamento e distribuição de protetores solares para agricultores, compras e prestação de contas por meio de processos judiciais contra o município e/ou Estado, entre outros.

\section{METODOLOGIA}

Este trabalho seguiu o método de relato de experiência. Trata-se de um relato e uma reflexão sobre uma atividade prática e colaborativa, com vistas à aplicação futura na Farmácia Municipal de Canguçu/RS. Os autores deste relato não se colocam apenas como observadores, mas como propositores e membros da equipe que elaborou o plano de intervenção na realidade da $\mathrm{AF}$ do município.

São apresentados os passos da construção de um PO, de acordo com o Planejamento Estratégico Situacional (PES) proposto por Carlos Matus, que tem por objetivo proporcionar direcionalidade 
à gestão, neste caso da Assistência Farmacêutica, a fim de intervir em uma realidade sobre a qual não é possível ter total controle.

O PES é composto por quatro momentos interdependentes: explicativo, normativo, estratégico e tático-operacional (Azevedo, 1992; Toni, 2004). O momento explicativo está relacionado ao entendimento do que se pretende realizar, das oportunidades e dos problemas que serão enfrentados. Nesta etapa também é realizada a Espinha de Peixe ou Diagrama de Ishikawa. De acordo com De Seta, Pepe e O'dwyer (2006), esta serve para evidenciar por meio de um gráfico a relação entre o resultado (efeito) e os fatores (causas) que o influenciam. O momento normativo está relacionado a todo o processo de planejamento, logo é a operação que pretende superar os problemas cruciais (nós críti$\cos$ ), levando à transformação da situação inicial em direção ao objetivo. O momento estratégico está relacionado à viabilidade de superar os obstáculos e alcançar o objetivo. O momento tático-operacional é a etapa decisiva do planejamento situacional que corresponde ao processo de tomadas de decisão (Azevedo, 1992; Toni, 2004).

As informações foram obtidas por meio de oficinas de construção do PO, pesquisa bibliográfica e anotações de um dos autores ao longo da vivência do processo. As oficinas, divididas em quatro etapas, ocorreram entre julho de 2014 e janeiro de 2015. Os dados foram analisados conjuntamente entre os autores.

Todos os atores envolvidos no processo de construção do $\mathrm{PO}$ assinaram o Termo de Consentimento Livre e Esclarecido para uso dos dados levantados durante o estudo proposto pelo curso de especialização em Gestão da Assistência Farmacêutica.

\section{RESULTADOS}

$\mathrm{Na}$ elaboração do $\mathrm{PO}$ foram desenvolvidas quatro oficinas, realizadas na sala de reuniões da Secretaria Municipal de Saúde de Canguçu/RS. As oficinas foram subdividas nos momentos descritos a seguir, de acordo com a sequência do Planejamento Estratégico Situacional.

O processo de planejamento para a elaboração do Plano Operativo incluiu a mobilização de diferentes atores que foram escolhidos devido as suas possíveis relações com a proposta do Plano Operativo (Quadro 1). A prefeitura de Canguçu possuía 259 funcionários lotados na SMS, dos quais 78 (30\%) servidores tinham nível superior. Para a realização do momento explicativo foram convidados 11 profissionais que representavam os setores mais relevantes para este estudo, mas compareceram apenas 7 . 
Quadro 1 - Quadro de atores que participaram da elaboração do PO

\begin{tabular}{|c|c|c|c|c|}
\hline ATORES & $\begin{array}{c}\text { TEMPO } \\
\text { ATUAÇÃO } \\
\text { NO SUS }\end{array}$ & IDADE & ATIVIDADE & JUSTIFICATIVA \\
\hline $\begin{array}{c}\text { Secretária Municipal } \\
\text { de Saúde }\end{array}$ & $\begin{array}{c}8 \text { anos e } 11 \\
\text { meses }\end{array}$ & 46 & Gestor da SMS & $\begin{array}{c}\text { Todas as possíveis mudanças necessitam } \\
\text { de seu aval }\end{array}$ \\
\hline $\begin{array}{l}\text { Farmacêutico Respon- } \\
\text { sável Técnico }\end{array}$ & $\begin{array}{c}8 \text { anos e } 11 \\
\text { meses }\end{array}$ & 35 & $\begin{array}{l}\text { Gestão da Farmácia Munici- } \\
\text { pal de Canguçu }\end{array}$ & $\begin{array}{c}\text { Encaminhamento de processos do com- } \\
\text { ponente especializado da Assistência } \\
\text { Farmacêutica }\end{array}$ \\
\hline Advogado & $\begin{array}{c}5 \text { anos e } 5 \\
\text { meses }\end{array}$ & 37 & $\begin{array}{l}\text { Assistência Judiciária da Pre- } \\
\text { feitura Municipal de Canguçu }\end{array}$ & $\begin{array}{c}\text { Analisa processos judiciais contra o mu- } \\
\text { nicípio }\end{array}$ \\
\hline Enfermeira & $\begin{array}{l}10 \text { anos e } 5 \\
\text { meses }\end{array}$ & 37 & $\begin{array}{l}\text { Diretora do Departamento de } \\
\text { Ações, Programas e Serviços } \\
\text { de Saúde }\end{array}$ & $\begin{array}{l}\text { Representa uma grande parcela dos usuá- } \\
\text { rios de medicamentos do componente } \\
\text { especializado }\end{array}$ \\
\hline Farmacêutico & $\begin{array}{l}4 \text { anos e } 9 \\
\text { meses }\end{array}$ & 33 & $\begin{array}{c}\text { Responsável pela Central de } \\
\text { Abastecimento Farmacêutico } \\
\text { (CAF) }\end{array}$ & $\begin{array}{c}\text { Conferência da medicação e distribuição } \\
\text { para as Unidades Básicas de Saúde }\end{array}$ \\
\hline $\begin{array}{l}\text { Auxiliar Administra- } \\
\text { tivo }\end{array}$ & 6 anos & 30 & $\begin{array}{l}\text { Responsável pelo Setor de } \\
\text { Agendamento de Consultas }\end{array}$ & $\begin{array}{l}\text { Encaminhamentos de exames de alta } \\
\text { e média complexidade, necessários à } \\
\text { abertura ou reavaliação dos processos } \\
\text { administrativos de medicamentos do } \\
\text { componente especializado da Assistência } \\
\text { Farmacêutica. }\end{array}$ \\
\hline $\begin{array}{l}\text { Auxiliar Administra- } \\
\text { tivo }\end{array}$ & $\begin{array}{c}23 \text { anos e } 3 \\
\text { meses }\end{array}$ & 45 & $\begin{array}{c}\text { Funcionária do Setor Finan- } \\
\text { ceiro }\end{array}$ & $\begin{array}{c}\text { Responsável pela conferência de verbas } \\
\text { para a compra de medicamentos }\end{array}$ \\
\hline $\begin{array}{l}\text { Auxiliar Administra- } \\
\text { tivo }\end{array}$ & 6 anos & 47 & $\begin{array}{l}\text { Responsável pelos depar- } \\
\text { tamentos Administrativo e } \\
\text { Financeiro da SMS }\end{array}$ & Coordenador da Farmácia Municipal \\
\hline Médico Prescritor & 4 anos e 1 mês & 35 & $\begin{array}{l}\text { Atendimento médico no } \\
\text { Pronto Atendimento }\end{array}$ & $\begin{array}{c}\text { Responsável pela maior parte das pres- } \\
\text { crições de medicamentos da Farmácia } \\
\text { Municipal }\end{array}$ \\
\hline
\end{tabular}

Fonte: Os autores.

\section{Momento Explicativo: Definição e priorização dos problemas}

Na reunião foi realizada uma dinâmica de grupo, ocasião em que todos explanaram suas ideias e sugestões até se chegar a um consenso geral. $\mathrm{O}$ grupo demonstrou interesse em participar do $\mathrm{PO}$ e colaborou com informações e conhecimentos relevantes na elaboração do momento explicativo. Não compareceram os representantes das áreas odontológica e enfermagem, pois estavam em horário de trabalho e não puderam ser dispensados. A ausência de alguns profissionais ocorreu pelo fato de terem sido convidados com um prazo muito curto até o dia da reunião, fazendo com que dispusessem de pouco tempo para se programar. A outra farmacêu- tica compareceu à oficina após a matriz de priorização de problemas, pois estava trabalhando, logo não foram computados os seus votos nesta etapa.

Os problemas elencados foram: (a) excessiva judicialização, (b) desatualização da Remume da Farmácia Municipal de Canguçu, (c) problemas na entrega dos medicamentos pela empresa fornecedora e (d) condicionalidades legais para aquisição de medicamentos.

Cada participante pontuou os problemas elencados quanto à magnitude, transcendência, vulnerabilidade, urgência e factibilidade. Ao final da reunião foi feita uma soma geral das notas de todos os atores envolvidos no processo de construção do Plano Operativo. Não houve conflito de ideias, apenas 
concepções diferentes em relação à nota atribuída. Todos concordaram desde o início quanto aos problemas citados em relação ao foco do PO.

Neste encontro, após a pontuação e com a definição do problema priorizado, foram identificados seus descritores, ou seja, as evidências que revelam que aquela situação priorizada é um problema.

A segunda fase do momento explicativo ocorreu com a construção da Espinha de Peixe; de posse dos descritores, do problema priorizado e da imagem-objetivo, foi elaborada uma apresentação da Espinha de Peixe para que todos os atores envolvidos pudessem emitir suas opiniões e sugestões. Não houve a necessidade de reescrever o problema nem os descritores, pois houve um consenso geral desde o início da primeira fase. Após a análise crítica e minuciosa do problema, descritores e imagem-objetivo, foram sendo apresentadas causas e suas respectivas consequências até se chegar à causa convergente: falta de comunicação entre os setores administrativo, financeiro, equipe técnica, compras e defensoria pública; e na sua respectiva consequência: aumento dos gastos com saúde.

\section{Momento Normativo}

O momento normativo contou com a participação da secretária municipal de Saúde, o farmacêutico responsável técnico pela $\mathrm{AF}$, uma auxiliar administrativa, responsável pelo setor financeiro, e um auxiliar administrativo, responsável pelo setor administrativo-financeiro. Como a reunião foi realizada em janeiro, período do ano em que muitos servidores se encontram em férias, não houve a participação da outra farmacêutica e do advogado, responsável pela assessoria jurídica da prefeitura de Canguçu. Em relação à área médica, o médico que participou da elaboração do momento explicativo não pôde participar desta etapa e das seguintes pelo fato de se encontrar licenciado por motivos de interesse particular. Após a realização desta etapa foi possível identificar os objetivos específicos, as operações e as ações a serem realizadas. Como objetivos, pode-se citar: promover a participação de diversos segmentos da área de saúde na construção da Remume a fim de estabelecer estratégias para reduzir a não adesão ou abandono do tratamento, o agravamento do quadro clínico e novas consultas ou internações e evitar gastos excessivos; otimizar a aquisição de medicamentos de acordo com o perfil epidemiológico do município, evitando o desperdício de verbas públicas; reduzir o número excessivo de ordens judiciais, a fim de diminuir os gastos extras com medicamentos não pertencentes à farmácia básica e elevar o nível de satisfação do usuário, evitando novos processos jurídicos. Foram definidas como operações necessárias para que os objetivos sejam alcançados: criação de uma Comissão de Farmácia e Terapêutica (CFT) para planejamento na seleção e compra de medicamentos; planejamento na aquisição de fármacos baseados no perfil epidemiológico da população; atualização da lista de remédios que compõem a Farmácia Básica levando em conta as necessidades dos usuários e adequação da Remume com base no perfil epidemiológico dos pacientes. Entre as ações necessárias estão: elaborar o projeto de instalação da CF; estudo da demanda mensal de prescrições atendidas; estudo das medicações dispensados por ordem judicial pelo município; adequação da aquisição de medicamentos conforme demanda investigada; levantamento daqueles de maior prevalência de uso pela população a partir dos cadastros individuais das USF, Caps e média complexidade; selecionar em reunião da CFT os fármacos que deverão ser incluídos na Remume e manter a regularidade de oferta a partir do planejamento e sistematização da compra.

\section{Momento Estratégico}

Nesta etapa fez-se uma análise da matriz do momento normativo para a elaboração da matriz do momento estratégico. Para tanto foi utilizado um computador ligado a um projetor, a fim de que fosse possível discutir acerca do que havia sido exposto na matriz do momento normativo e complementar o quadro do momento estratégico. Partindo-se das operações e ações elencadas no momento anterior, foram realizadas nesta etapa as análises de viabilidade e factibilidade, o déficit e a atividade estratégica para cada operação. 


\section{Momento Tático-Operacional}

A matriz do momento tático-operacional também foi elaborada no mês de janeiro e contou com a participação dos mesmos atores envolvidos na elaboração do momento estratégico. Fez-se uma análise de cada objetivo específico, operações e ações da matriz do momento estratégico para a elaboração da matriz final do Plano Operativo, inserindo-se os recursos financeiros, caso necessário ou estimado, a responsabilidade e centralidade (ator principal: órgão ou setor ou técnico) e outros parceiros (órgão ou setor ou técnico), assim como o prazo para realização das ações e o indicador de avaliação (operação). Constatou-se a necessidade de recursos no valor de $\mathrm{R} \$ 20.000,00$ ao mês para a aquisição de medicamentos que não pertençam à Farmácia Básica. Como indicadores de avaliação é possível citar: decreto de criação da CFT, projeto de Lei da Remume atualizada, número absoluto de medicamentos solicitados por ordem judicial/mês e razão entre a relação de medicamentos definidos na Remume e a relação de remédio em estoque na Farmácia Municipal/mês.

Os aspectos positivos percebidos nas quatro oficinas foram a troca de conhecimento entre os diversos atores, o que enriqueceu o trabalho sobre o Plano Operativo, além da receptividade de todos na busca de novos conhecimentos e possíveis melhorias em relação à Assistência Farmacêutica. Como aspectos negativos cita-se a ausência de alguns colegas, que poderiam ter contribuído para uma excelente troca de experiências.

O monitoramento e avaliação do planejamento são importantes para que seja possível acompanhar a execução do plano, seu desenvolvimento e suas transformações, possibilitando que ele seja atualizado quando se fizer necessário. Como potencialidades desta ferramenta menciona-se a organização da equipe de trabalho, a atuação dos diferentes atores, a definição do foco de atuação e o fato de este ser um processo dinâmico que pode sofrer atualizações sempre que for preciso. Conforme Toni (2004), a análise de problemas, a identificação de cenários, a visualização de atores e o enfoque estratégico são partes fundamentais e que distinguem o PES em relação a outros métodos de planejamento.

No que se refere às fragilidades, evidenciou-se a não permanência de alguns atores envolvidos no processo de elaboração do Plano Operativo, que necessitaram deixar a equipe por afastamento do serviço ou por motivo de férias. É possível prever também que o déficit em tecnologia de informação em algumas Unidades Básicas de Saúde venha ser uma fragilidade na obtenção de dados para este estudo.

\section{DISCUSSÃO}

Os desafios para que a Assistência Farmacêutica seja instituída e estruturada de forma efetiva na esfera municipal, especialmente na Atenção Básica à Saúde (ABS), iniciam-se pela conscientização, por parte dos gestores, da importância de uma estrutura física adequada, da capacitação dos funcionários e da organização dos processos, tornando o acesso da população aos medicamentos mais racional e eficiente (Oliveira; Assis; Barboni, 2010).

Acredita-se que o plano descrito neste texto será eficaz se a alta direção (gestão da prefeitura e Secretaria Municipal de Saúde) participar de todas as atividades previstas, pelo tempo necessário até a execução de todos os momentos que compõem o PES. É necessário lembrar que a cada quatro anos a gestão municipal passa por mudanças, o que dificulta a continuidade dos projetos e programas de saúde do município.

A perspectiva é de que o PO seja aplicado assim que houver uma cobertura de USF que abranja todas as regiões da cidade. No momento do planejamento, a área coberta pelas Unidades de Saúde da Família ainda correspondia a 32,22\% do município (Departamento de Atenção Básica, 2015) e 50,51\% de cobertura de Unidades Básicas de Saúde (Sistema de Pactuação de Indicadores do Pacto pela Saúde, 2015), com projetos de ampliação do número de USF no interior do município e no aumento 
do atendimento na atenção básica que continuam em andamento na Secretaria Municipal de Saúde de Canguçu.

Inicialmente havia o entendimento de que a judicialização de processos seria o principal problema. Após a elaboração do PO, contudo, houve a constatação de que a desatualização da Remume da Farmácia Municipal de Canguçu/RS, por não estar de acordo com o perfil epidemiológico dos cidadãos canguçuenses, estaria elevando o número de processos judiciais contra o Estado e/ou município. De acordo com o sistema de Administração de Medicamentos do Estado (AME) do Rio Grande do Sul, em 2015 Canguçu possuía 665 pacientes com processos judiciais deferidos contra o Estado e/ou município. Neste sistema não consta nenhum processo judicial deferido somente contra o município. No ano de 2014 foi gasto com processos judiciais o valor de R\$3.951,50 e em 2015, até o mês de junho, a despesa foi de $\mathrm{R} \$ 5.577,94$.

\section{CONCLUSÃ̃}

Este trabalho teve a intenção de relatar uma experiência de elaboração de um Plano Operativo para a Farmácia Municipal de Canguçu/RS, detalhando todas as etapas do processo. Tornou-se, contudo, mais do que um relato, uma reflexão a respeito de todos os dilemas, processos e obstáculos que envolvem a construção de um PO, o foco central do planejamento, os seus objetivos e o espaço no qual ele deve ser aplicado. Isto é o que se denomina Educação Permanente em Saúde. A integração ensino-serviço que oportuniza problematizar a prática com vistas à melhoria do Sistema Único de Saúde.

Salienta-se também a participação ativa e o interesse de toda a equipe de trabalho durante todas as oficinas de elaboração do PO, pois entende-se que um ator com conhecimento aprofundado do problema pode enfrentá-lo com muito mais facilidade.

Esta experiência foi importante para a equipe constatar que as despesas da Secretaria Municipal de Saúde com os processos judiciais de medicamen- tos estavam relacionadas com a desatualização da Remume. Ao contrário do que se pensava, a judicialização não é um problema isolado, mas proveniente de outros agravantes.

Com base no que foi exposto, espera-se que o presente estudo possa contribuir para a qualificação da Assistência Farmacêutica na Atenção Básica do município com o uso diário da ferramenta Planejamento Estratégico Situacional na identificação de problemas, com a participação dos diversos atores envolvidos no atendimento da Secretaria Municipal de Saúde.

Deseja-se que o PES possa ser posto em prática o mais breve possível, a fim de que os problemas gerados pela desatualização da Remume, como a judicialização de processos solicitando medicamentos, possam ser resolvidos ou amenizados.

\section{REFERÊNCIAS}

\section{ADMINISTRAÇÃO DE MEDICAMENTOS DO} ESTADO. AME. Disponível em: <https://secweb. procergs.com.br/ame/ame/Interface/Html/index. jsp>. Acesso em 18 jun. 2015.

ARAÚJO, Aílson et al. Perfil da assistência farmacêutica na atenção primária do Sistema Único de Saúde. In: Ciência \& Saúde Coletiva, Rio de Janeiro, p. 611-617, 2008. Disponível em: <http://www.scielo.br/scielo. php?pid=S1413-81232008000700010\&script $=$ sci abstract\&tlng $=$ pt $>$. Acesso em: 27 jun. 2015.

ARTMANN, Elizabeth. O planejamento estratégico situacional no nível local: um instrumento a favor da visão multissetorial. In: Cadernos da Oficina Social 3: Série Desenvolvimento Local, Rio de Janeiro: Coppe/UFRJ, 2000. Disponível em: $<$ http://www.scielosp.org/scieloOrg/php/reflinks. php?refpid=S1413-8123201100030002300010\&pi $\mathrm{d}=\mathrm{S} 1413-81232011000300023 \& \operatorname{lng}=\mathrm{en}>$. Acesso em: 7 jun. 2015.

ATLAS BRASIL. Atlas do Desenvolvimento Humano no Brasil. Canguçu, RS. 2010. Disponível em: $<$ http://www.atlasbrasil.org.br/2013/pt/perfil_m/ cangucu_rs $>$. Acesso em: 22 maio 2015. 
AZEVEDO, C. da S. Planejamento e gerência no enfoque estratégico-situacional de Carlos Matus. In: Caderno de Saúde Pública, Rio de Janeiro, 1992. Disponível em: <http://www.scielo.br/pdf/csp/ v8n2/v8n2a03>. Acesso em: 23 maio 2015.

BRASIL. Constituição da República Federativa do Brasil de 1988. Brasília, 1988. Disponível em: $<$ http://www.planalto.gov.br/ccivil_03/constituicao/ ConstituicaoCompilado.htm>. Acesso em: 25 jun. 2015.

. Lei ${ }^{0} 8.080$, de 19 de setembro de 1990. Dispõe sobre as condições para a promoção, proteção e recuperação da saúde, a organização e o funcionamento dos serviços correspondentes e dá outras providências. Brasília, 1990. Disponível em: $<$ http://www.planalto.gov.br/ccivil_03/Leis/L8080. htm>. Acesso em: 25 jun. 2015.

Ministério da Saúde. Secretaria de Saúde. Departamento de Atenção Básica. Política Nacional de Medicamentos. Brasília, 2001. Disponível em: $<$ http://bvsms.saude.gov.br/bvs/publicacoes/politica_medicamentos.pdf $>$. Acesso em: 15 jun. 2015.

Ministério da Saúde. Resolução no 338, de 6 de maio de 2004. Dispõe sobre a Politica Nacional de Assistência Farmacêutica. Brasília, 2004. Disponível em: <http://bvsms.saude.gov.br/bvs/ saudelegis/cns/2004/res0338_06_05_2004.html>. Acesso em: 27 jun. 2015.

Ministério da Saúde. Portaria n 204 de 29 de janeiro de 2007. Regulamenta o financiamento e a transferência dos recursos federais para as ações e os serviços de saúde, na forma de blocos de financiamento, com o respectivo monitoramento e controle. Brasília: 2007. Disponível em: $<\mathrm{http}$ // bvsms.saude.gov.br/bvs/saudelegis/gm/2007/ prt0204_29_01_2007.html >. Acesso em: 20 jun. 2015.

Ministério da Saúde. Portaria ${ }^{0} 1.554$ de 30 de julho de 2013. Dispõe sobre as regras de financiamento e execução do Componente Especializado da Assistência Farmacêutica no âmbito do Sistema Único de Saúde (SUS). Brasília, 2013. Disponível em: <http://bvsms.saude.gov.br/bvs/ saudelegis/gm/2013/prt1554_30_07_2013.html>. Acesso em: 20 jun. 2015.
Ministério da Saúde. Relação Nacional de Medicamentos Essenciais - Rename 2014. Brasília, 2014. Disponível em: $<$ http://www.youblisher. $\mathrm{com} / \mathrm{p} / 1138570-R E N A M E 2014$. Acesso em: 20 jun. 2015.

DEPARTAMENTO DE ATENÇÃO BÁSICA (DAB). Disponível em: <http://dab.saude.gov.br/ portaldab/historico_cobertura_sf.php $>$. Acesso em: 9 jul. 2015.

DE SETA, M. H.; PEPE, V. L. E.; O’DWYER, G. (Org.). Gestão e vigilância sanitária: modos atuais de pensar e fazer. Rio de Janeiro: Fiocruz, 2006. Disponível em: <https://books.google.com.br/ books?hl=pt-BR\&lr=\&id=MOTpAgAAQBAJ\&oi $=$ fnd\&pg $=$ PA $167 \& d q=$ espinha $+\mathrm{de}+$ peixe + carlos + matus\&ots $=$ SHqLGEPgdw\&sig $=\mathrm{jxd} 8 \mathrm{u} 6 \mathrm{dUhS} 72 \mathrm{dX}$ G5ciTFCZuj42I $\# \mathrm{v}=$ onepage $\& \mathrm{q} \& \mathrm{f}=$ false $>$. Acesso em: 28 jun. 2015.

FREDES, Mara Elis Ferreira. Judicialização do acesso a medicamentos: perfil dos usuários residentes no município de Pelotas/RS. 2012. $52 \mathrm{f}$. Monografia (Especialização em Gestão em Saúde) - Universidade Federal do Rio Grande do Sul, Porto Alegre, 2012.

INSTITUTO BRASILEIRO DE GEOGRAFIA E ESTATÍSTICA. Cidades. Rio Grande do SulCanguçu. 2013. Disponível em: < http://ibge.gov. $\mathrm{br} /$ cidadesat/xtras/home.php $>$. Acesso em: 22 maio 2015.

OLIVEIRA, Luciane; ASSIS, Marluce; BARBONI, André. Assistência farmacêutica no Sistema Único de Saúde: da Política Nacional de Medicamentos à Atenção Básica à Saúde. In: Ciência \& Saúde Coletiva, Rio de Janeiro, v. 15, n.3, p. 3.561-3.567, 2010. Disponível em: $<$ http:// www.scielo.br/scielo.php?script=sci_arttext\&pi $\mathrm{d}=\mathrm{S} 1413-81232010000900031>$. Acesso em: 27 jun. 2015.

ORGANIZACIÓN PANAMERICANA DE LA SALUD; ORGANIZACIÓN MUNDIAL DE LA SALUD. OPS; OMS. Servicios farmacéuticos basados en la atención primaria de salud. Washington, DC, 2013. Disponível em: <http://www.paho. org/hq/index.php?option $=$ com_docman\&task $=$ doc view\&gid $=22480 \&$ Itemid $=>$. Acesso em: 27 jun . 2015. 
PEPE, Vera Lúcia Edais et al. A judicialização da saúde e os novos desafios da gestão da assistência farmacêutica. In: Ciência \& saúde coletivam, Rio de Janeiro, v. 15, n. 5, p. 2.405-2.414, 2010. Disponível em: <http://www.scielo.br/ scielo.php?script $=$ sci_arttext\&pid $=$ S 1413 $-81232010000500015 \& \operatorname{lng}=\mathrm{en} \& \mathrm{nrm}=\mathrm{iso}>$. Acesso em: 19 jun. 2015.

RIO GRANDE DO SUL. Secretaria Estadual de Saúde do Estado do Rio Grande do Sul. Documentos e exames necessários para solicitação administrativa de medicamentos do componente especializado da assistência farmacêutica. Porto Alegre, 2015. Disponível em: <http://www.saude.rs.gov.br/ upload/20150617152554documentos_e_exames_ para_solicitacao_administrativa_de_medicamentos_do_ceaf_2015_versao_junho_de_2015_ word_- 97.pdf $>$. Acesso em: 20 jun. $201 \overline{5}$ a.

Secretaria Estadual de Saúde do Estado do Rio Grande do Sul. Componente Especializado da Assistência Farmacêutica. Porto Alegre, 2015. Disponível em: <http://www.saude.rs.gov.br/lista/153/ Componente_Especializado $>$. Acesso em: 13 jul. $2015 b$.

Secretaria Estadual de Saúde do Estado do Rio Grande do Sul. Resolução $n^{\circ}$ 2016/14-CIB/RS. Porto Alegre, 2014. Disponível em: <http://www. saude.rs.gov.br/upload/20140520152042cib_216. pdf $>$. Acesso em: 20 jun. 2015.

RIVERA, Francisco Javier Uribe; ARTMANN, Elizabeth. Planejamento e gestão em saúde: flexibilidade metodológica e agir comunicativo. In: Ciência \& Saúde Coletiva. Rio de Janeiro, v. 15, n. 5, p. 355-365, 1999. Disponível em: <http://www. scielo.br/scielo.php?script=sci_arttext\&pid=S1413$-81231999000200010 \& \operatorname{lng}=\mathrm{en} \& \mathrm{nrm}=\mathrm{iso}>$. Acesso em: 19 jun. 2015.

SISTEMA DE PACTUAÇÃO DE INDICADORES DO PACTO PELA SAÚDE (SISPACTO). Disponível em: <http://aplicacao.saude.gov.br/sispacto/ faces/login.jsf $>$. Acesso em: 9 jul. 2015.

TONI, Jackson. O que é o Planejamento Estratégico Situacional. In: Revista Espaço Acadêmico, Maringá: Universidade Estadual de Maringá, n. 32, jan. 2004. Disponível em: $<$ http://www.espacoacademico.com.br/032/32ctoni.htm>. Acesso em: 23 maio 2015.
VIEIRA, Fabiola Sulpino; ZUCCHI, Paola. Distorções causadas pelas ações judiciais à política de medicamentos no Brasil. Revista de Saúde Pública. São Paulo, 2007. p. 214-222. Disponível em: <http:/www.scielo.br/scielo.php?script=sci arttext\&pid=S0034-89102007000200007\&lng=en $\&$ nrm=iso > . Acesso em: 19 jun. 2015.
Recebido em: 16/11/2015

Aceito em: 29/12/2015 